Urologe 2016 · 55:1329-1334

DOI 10.1007/s00120-016-0078-5

Online publiziert:31. Mai 2016

(c) The Author(s) 2016. This article is available

at SpringerLink with Open Access

CrossMark

\author{
H. H. Dahm ${ }^{1}$ - V. Lehnen-Holtum ${ }^{2}$ H. Rübben ${ }^{3}$ \\ ${ }^{1}$ Institut für Pathologie, Esslingen, Deutschland \\ ${ }^{2}$ Praxis Ratheim, Ratheim, Deutschland \\ ${ }^{3}$ Klinik und Poliklinik für Urologie, Essen, Deutschland
}

\title{
Karyometrie BBN-induzierter Präkanzerosen des Urothels
}

\section{Eine experimentelle Analyse}

\section{Hintergrund}

Im Tierversuch diente das durch N-Butyl-N-(4-Hydroxybutyl)nitrosamin $(\mathrm{BBN})$ induzierte Karzinom der Harnblase häufig als Modell zur Untersuchung der Kanzerogenese oder zu Therapieversuchen $[19,23]$.

$\mathrm{N}$-[4-(5-nitro-2-furyl)-2-thiazolyl]formamid (FANFT) und N-Methyl-N-Nitrosoharnstoff(MNU) wurden gleichfalls als Karzinogene verwandt. Als Folge der Kanzerogenexposition wurde zunächst eine Hyperplasie des Urothels beschrieben $[1,2,4-6,8-12,14-16,18,22,23]$. Ferner wurden papilläre oder noduläre Hyperplasien und Papillome beobachtet [2, 4-6, 8, 15, 18, 22, 23]. Schichtungsund Reifungsstörungen des Urothels wurden als leichte, mittelschwere oder schwere Hyperplasie oder auch als Dysplasie und schließlich als Carcinoma in situ bezeichnet $[4-6,8,9,12,14-16,22$, 23]. Die Diagnosen basierten auf keiner verbindlichen Klassifikation.

Ziel dieser Arbeit war, die BBNinduzierten morphologischen Veränderungen des Urothels auf der Grundlage karyometrischer Maße an Ausstrichpräparaten als Basis weitergehender Untersuchungen objektiv darzustellen.

\section{Material und Methoden}

Präkanzerosen des Urothels der Harnblasen von 60 weiblichen Wistar-Ratten (Landeszuchtanstalt Hannover) wurden durch N-Butyl-N-(4-Hydroxybutyl)nitrosamin (BBN; Prof. Dr. R. Preuss- mann, Deutsches Krebsforschungszentrum, Heidelberg) in 0,05 Vol.-\%iger Lösung als Trinkwasser induziert. Jeweils 3 Tiere wurden in einem Makrolonkäfig (Ehret, Emmendingen) gehalten. Das Gewicht betrug zu Versuchsbeginn 180-200 g. Standardfutter (Eggersmann, Rinteln) und BBN-Lösung wurden ad libitum verfüttert. Die Raumtemperatur betrug $22^{\circ} \mathrm{C}$, die relative Luftfeuchtigkeit $55 \%$, der Hell-Dunkel-Rhythmus 12 h. 6 Kontrolltiere tranken Leitungswasser. Nach einer BBN-Gabe von 2-20 Wochen, jeweils gefolgt von einem 2-wöchigen BBN-freien Intervall, wurden 2-wöchentlich 6 Tiere in Äthernarkose durch intraperitoneal in Überdosierung appliziertes Nembutal (Abbott, Ludwigshafen) getötet. Bei 3 Tieren wurde das Urothel von der Lamina propria abgelöst und aufObjektträgern ausgestrichen. Die Ausstriche wurden mit MerckofixSpray (Merck, Darmstadt) fixiert und nach Papanicolaou gefärbt. Für die vergleichende histologische Untersuchung wurden 3 Tiere mit einer gepufferten Formalin-Glutaraldehyd-Lösung perfusionsfixiert. Die zytologischen Präparate wurden mit 1000facher Vergrößerung fotografiert. Auf Papierabzügen mit der Endvergrößerung $\times 2100$ wurden die Zellkerne mit einem Cursor umfahren. Von den Kontrollen und 2-Wochen-Gruppen wurden 300-600 Kerne ausgemessen. Das Messsystem MOPAMO2 (Kontron, Eching) war an den Rechner HP 9825A (Hewlett-Packard, Böblingen) angeschlossen, der Umfang, Fläche, maximalen Durchmesser, Halb- achsenrelation und Formfaktor sowie die jeweiligen Maximal- und Minimalwerte, Durchschnitt und Standardabweichung ausgab. Die ermittelten Maße wurden in 8-10 Klassen unterteilt und im $\chi^{2}$-Test geprüft.

\section{Ergebnisse}

\section{Histologie}

Das Übergangsepithel der Kontrolltiere umfasste je eine Schicht von Superfizial-, Intermediär- und Basalzellen. Nach 2 Wochen BBN war das Epithel fokal durch eine Vermehrung der Basalund Intermediärzellen leicht verbreitert. In Woche 4 waren die ovalären Kerne der vermehrten Intermediärzellen mit der Längsachse senkrecht zur Basalmembran ausgerichtet. Vereinzelte leichte Kernvergrößerungen erweckten den Verdacht auf eine leichte Dysplasie. Das Epithel umfasste in Woche 6 überwiegend eine von Feld zu Feld unterschiedlich dicke Zellschicht mit zumeist leicht, vereinzelt auch deutlich vergrößerten Kernen und dem Bild einer Dysplasie. Eine leichte bis mäßige Dysplasie, teilweise auch in einer Plattenepithelmetaplasie, fand sich in Woche 8. Die Dysplasieherde waren in Woche 10 stärker ausgedehnt. In Woche 12 herrschte in der Harnblase insgesamt eine leichte bis mäßige Dysplasie vor. Normales Urothel war weitgehend geschwunden. Kleinherdige stärkere Atypien in Urothel und metaplastischem Plattenepithel kennzeichneten Woche 14. Im weiteren Verlauf des 


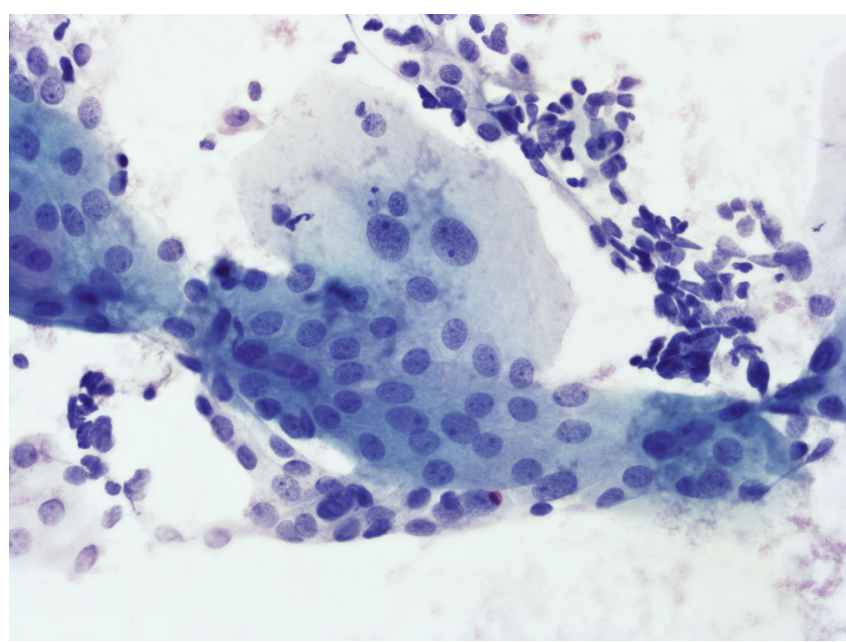

Abb. 1 A Kontrolle: dreischichtiges Urothel (Papanicolaou, Originalvergr. 400:1)

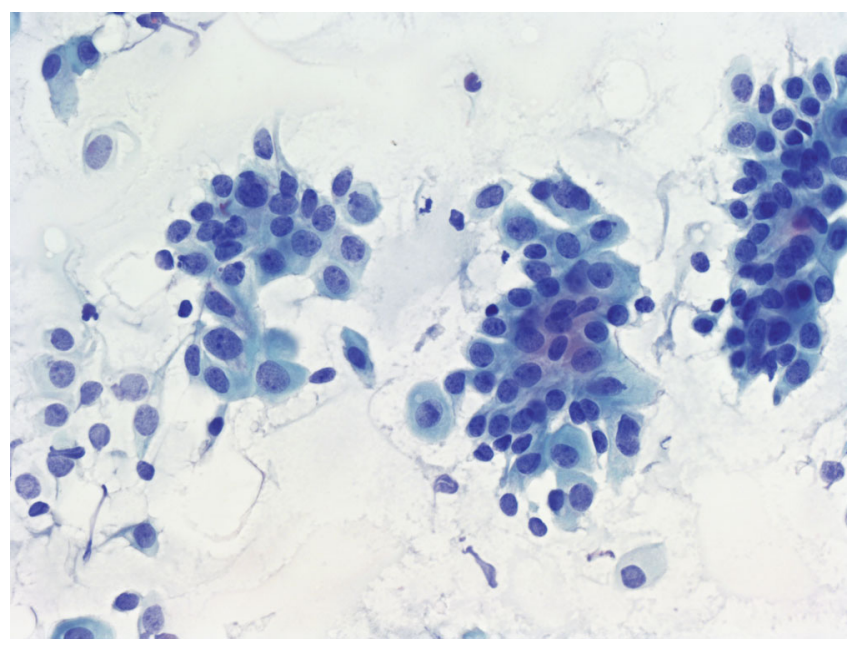

Abb. 3 \& N-butyl-N-(4-hydroxybutyl)nitrosamin Woche 8: mittelgradige Atypien des Urothels (Papanicolaou, Originalvergr. 400:1)

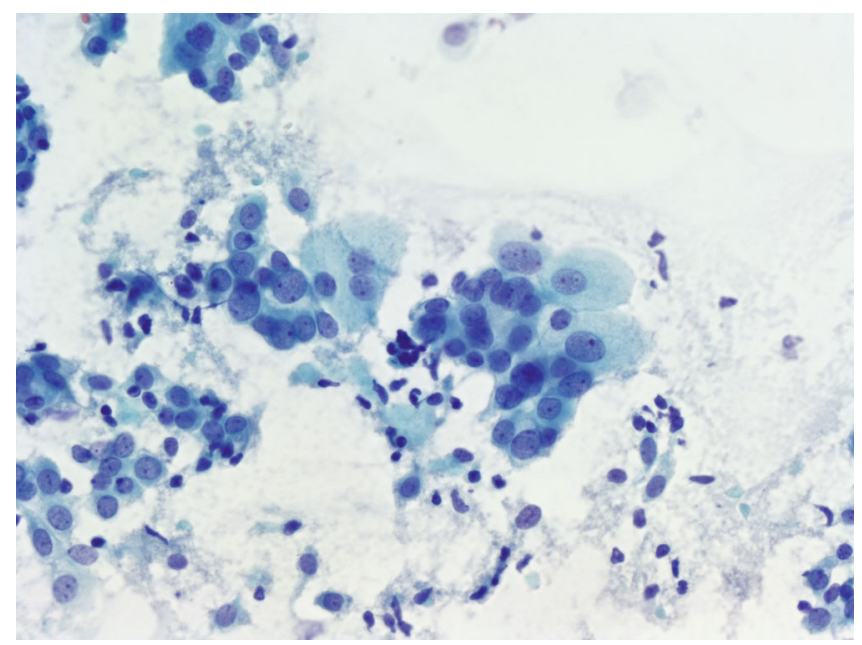

Abb. 2 ム N-butyl-N-(4-hydroxybutyl)nitrosamin Woche 2: Superfizialzellen mit verkleinertem Zytoplasma. Kernvergrößerung der Intermediärzellen (Papanicolaou, Originalvergr. 400:1)

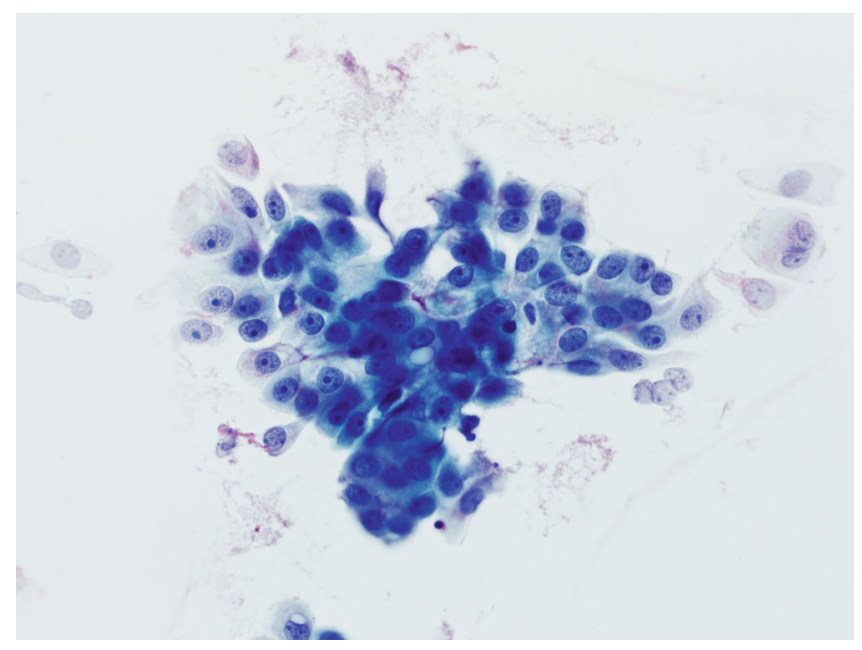

Abb. $4 \Delta$ N-butyl-N-(4-hydroxybutyl)nitrosamin Woche 20: schwere Atypien des Urothels (Papanicolaou, Originalvergr. 400:1)
Experiments bis zur Woche 20 nahm die Zahl von Zellen mit einer mäßigen oder auch schweren Kernanaplasie zu, wobei das Urothel überwiegend nur wenige Zellschichten aufwies und lediglich fokal verdickt war. Eine schwere Kernanaplasie des Urothels fand sich in papillomatösen Tumoren der Woche 20.

\section{Zytologie}

In den Ausstrichpräparaten des Urothels der Kontrolltiere waren die Superfizialzellen durch ein großes Zytoplasma gekennzeichnet und enthielten bläßchenförmige Kerne. Kerne und Zytoplasma der Intermediär- und Basalzellschicht waren deutlich kleiner (• Abb. 1). In den frühen Stadien waren vereinzelt solche normalen Epithelverbände erhalten. In Woche 2 war das Zytoplasma der Superfizialzellen leicht verkleinert (- Abb. 2). In Woche 6 waren die Kerne der Superfizialzellen verkleinert, die der Basal- und Intermediärzellen vergrößert und vereinzelt entrundet. Nukleolen waren vermehrt und teilweise vergrößert. In Woche 8 fand sich eine deutliche Anisokaryose der Intermediärschicht mit einer Zunahme des Dysplasiegrades (• Abb. 3). Zellen einer mäßigen Dysplasie des Urothels fanden sich in Woche 10. Bis zur Woche 20 nahm die Atypie des Epithels zu mit verstärkter
Kernpolymorphie, grobkörnigem Chromatin und prominenten, entrundeten Nukleolen (• Abb. 4).

\section{Karyometrie und Statistik}

Im $\chi^{2}$-Test der karyometrisch ermittelten Maße unterschieden sich die 10 BBN-exponierten Gruppen signifikant von der Kontrollgruppe und zumeist auch voneinander. Die Signifikanz der Trennung der Gruppen beruhte im Wesentlichen auf der Veränderung der Kernflächenmaße. Aus diesem Grund wurden im Folgenden ausschließlich diese Maße erörtert. Die Ergebnisse wurden wie folgt dargestellt. - Tab. 1 gibt das Kernflächen- 
maximum $\left(\mathrm{U}_{\mathrm{MAX}}\right)$, - $\operatorname{minimum}\left(\mathrm{U}_{\mathrm{MIN}}\right)$ sowie -mittelwert $\left(\mathrm{U}_{\mathrm{MIT}}\right)$ und -standardabweichung $\left(\mathrm{U}_{\mathrm{STDEV}}\right)$ an. Die ermittelten Kernflächenmaße wurden in 10 Klassen aufgeteilt. - Tab. 2 stellt die Größe der Klassen in $\mu \mathrm{m}^{2}$ und deren Häufigkeitssummen in den Intervallen dar. Zum besseren Vergleich wurden diese Häufigkeitssummen in einem Histogramm gewichtet (• Abb. 5).

Ziel der weiteren Analyse war, die Veränderung der Klassenverteilung zu ermitteln und mit den morphologischen Befunden $\mathrm{zu}$ verknüpfen.

Nach der 2-wöchigen BBN-Exposition unterschied sich die Verteilung der Kernflächenklassen signifikant von der Kontrollgruppe durch eine Abnahme in Größenklasse 4 und eine Zunahme in Klasse 7. Von Woche $2 \mathrm{zu}$ Woche 4 folgte eine signifikante Verschiebung von den Größenklassen 2-4 zu den Klassen 6 und 7. Von Woche $4 \mathrm{zu}$ Woche 6 folgte die Umkehrung des Trends mit Verschiebung von den Klassen 6-8 zu den Klassen 3 und 4 mit der kleineren Fläche. Es folgte sodann eine Umverteilung der Häufigkeiten innerhalb der Größenklassen, die offenbar nicht linear verlief, die von der 10. zur 12. Woche jedoch mit einer signifikanten Verschiebung zu den Klassen mit der größeren Kernfläche einherging. Veränderungen in den beiden Wochen 14 und 16 waren erkennbar, jedoch nicht signifikant. Von Woche 18 $\mathrm{zu}$ Woche $20 \mathrm{kam}$ es zur signifikanten Zunahme der kleineren Größenklassen 3 und 4 und einer Abnahme der Klassen 7 und 8 .

\section{Diskussion}

Die Karyometrie wurde zur Objektivierung des Malignitätsgrades von Harnblasentumoren bereits 1922 eingeführt [21]. Spätere Untersuchungen ergaben eine gute Korrelation zwischen der morphometrischen und der histologischen Klassifikation von klinischen Blasentumoren $[3,7,17]$. In der Literatur fanden sich keine karyometrischen Untersuchungen experimenteller Harnblasentumoren oder ihrer Vorstadien.

Ziel dieser Arbeit war, die BBNinduzierten morphologischen Veränderungen des Urothels auf der Grundlage

Urologe 2016·55:1329-1334 DOI 10.1007/s00120-016-0078-5

(c) The Author(s) 2016

\section{H. H. Dahm · V. Lehnen-Holtum · H. Rübben}

\section{Karyometrie BBN-induzierter Präkanzerosen des Urothels. Eine experimentelle Analyse}

Zusammenfassung

Hintergrund. Die Morphologie der experimentellen Präkanzerose der Harnblase wurde in der Literatur unterschiedlich interpretiert. Fragestellung. Ziel dieser Arbeit war, die Entwicklung der Präkanzerose des Urothels im zeitlichen Verlauf des Experiments vergleichend zytologisch und karyometrisch zu beschreiben.

Material und Methoden. Urotheliale Präkanzerosen wurden bei 60 Wistar-Ratten durch eine 0,05 Vol.-\%ige Lösung von $\mathrm{N}$-Butyl-N-(4-Hydroxybutyl)nitrosamin (BBN) als Trinkwasser induziert. Ab Woche 2 der BBN-Exposition wurden 6 Tiere in Intervallen von 2 Wochen bis zur Woche 20 getötet. Das Urothel von jeweils 3 Harnblasen wurde an Ausstrichpräparaten zytologisch und karyometrisch untersucht.

Ergebnisse. Die BBN-Exposition führte karyometrisch zu einem signifikanten
Unterschied der Kontrollgruppe von den BBN-exponierten 2-Wochen-Gruppen und dieser 2-Wochen-Intervalle. Der Unterschied bestand vorwiegend in einer signifikanten Verschiebung der Größenklassen der Kernflächenmaße.

Schlussfolgerungen. Morphologie und Karyometrie wiesen darauf hin, dass die biologisch entscheidenden Schritte der BBN-induzierten Präkanzerose wesentlich früher eingetreten waren als in der Literatur angenommen. Die Karyometrie bot eine objektive Basis der Beschreibung der experimentellen Präkanzerose des Urothels und ergänzender Untersuchungen.

Schlüsselwörter

Urothel - Kanzerogenese - Karyometrie . Zytologie $\cdot$ Hyperplasie

\section{Karyometry of BBN-induced precancerosis of the urothelium. An experimental analysis}

\section{Abstract}

Background. The morphology of experimental precancerous lesions of the urinary bladder has been interpreted quite differently by various authors.

Objectives. The aim of this investigation was to quantify these lesions by karyometry and, thus, to gain a more reliable understanding of the process.

Materials and methods. A total of 60 Wistar rats were fed with N-butyl-N-(4-hydroxybutyl)nitrosamine (BBN) at a concentration of $0.05 \%$ in their drinking water to induce preneoplastic changes of the urothelium. After the second week of BBN exposition, 6 animals were killed every 2 weeks up to week 20. Smears of the scraped off urothelium of 3 urinary bladders of each group were analyzed cytologically and karyometrically. Results. BBN exposition led to statistically significant changes of the karyometric values using the $x^{2}$ test to differentiate the control animals from the ones that had ingested BBN and the 2-week groups from each other. These changes consisted mainly in significant deviations of the size of the nuclear area within the different groups.

Conclusion. Morphological and karyometrical analysis showed that biologically relevant stages in the development of chemically induced urothelial precancerous lesions could be realized much earlier than had been assumed in recent publications. Karyometric analysis offered a valid basis to describe the early morphologic alterations of carcinogenesis.

\section{Keywords}

Urothelium · Carcinogenesis · Karyometry . Cytology $\cdot$ Hyperplasia karyometrischer Maße an Ausstrichpräparaten objektiv zu beschreiben.

Leichtgradige zytologische Veränderungen des Urothels waren in dem hier vorgestellten Experiment bereits nach einer 2-wöchigen BBN-Exposition festzu- stellen, die mit einer signifikanten Änderung der Kernflächenverteilung einhergingen. Kernunregelmäßigkeit und die Verschiebung zu den Klassen mit größerer Kernfläche nahmen nach 4 Wochen BBN signifikant zu. Nach 6 Wochen BBN 


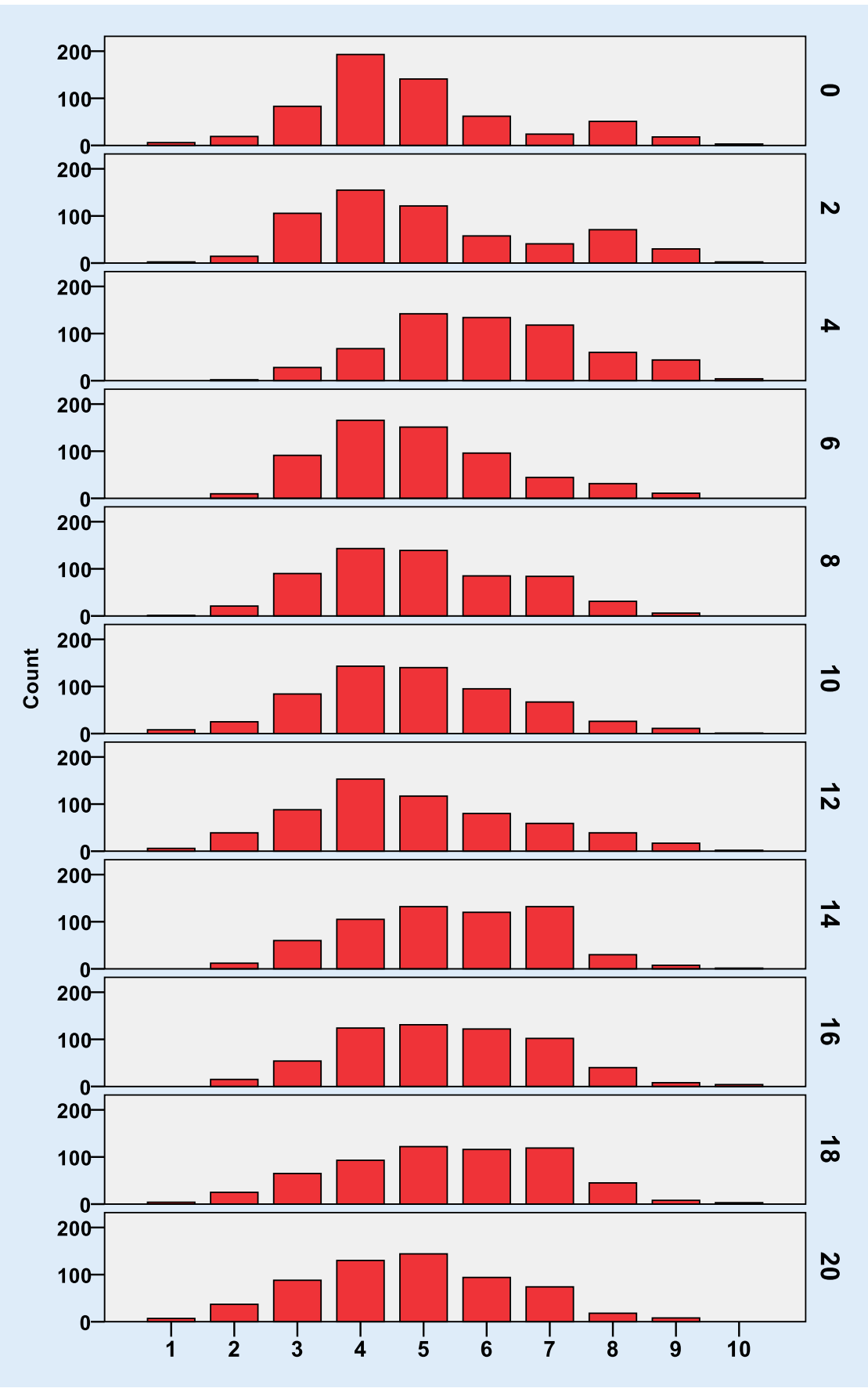

Abb. 5 ॥ Histogramm der Klassenverteilung

bestand histologisch bereits eine fokale Schichtungsstörung des Urothels, während sich die Kernflächen von den Klasse 6-8 zu Klassen 3 und 4, möglicherweise als Folge der Reifungsstörung des Urothels, verkleinerten. Insgesamt kam es im Verlauf von 14 Wochen jedoch zu einer signifikanten Zunahme der größeren Kernflächen, während sich zytologisch eine deutliche Anisokaryose entwickelte.
Eine Reifungsstörung des Epithels im Sinne einer Dysplasie war somit bereits nach einer 4-wöchigen, deutlicher nach einer 6-wöchigen BBN-Exposition anzunehmen, die zytologisch und karyometrisch im Verlauf des Experiments an Schweregrad zunahm.

Die formale Pathogenese des experimentellen Harnblasenkarzinoms wurde in der Literatur unterschiedlich beschrie- ben. Wie in der vorgelegten Arbeit wurde vielfach $\mathrm{BBN}$ in 0,05\%iger Lösung als Kanzerogen im Trinkwasser verfüttert $[1,2,6,9,10,12,14-16,18,23]$. Andere Arbeitsgruppen setzten FANFT $[4,22]$ oder MNU [8] ein. Als erste Reaktion fanden die Autoren eine Hyperplasie des Urothels [1, 2, 4, 6, 8-10, 12, 14-16, $18,22,23]$, in der Schichtungs- und Reifungsstörungen auftreten konnten $[6,8$, $9,12,14,15,22]$ und die als leichte, mittelschwere oder schwere Hyperplasie [4] beurteilt wurden. Die Veränderungen wurden auch als Dysplasie $[14,16]$ und schließlich als Carcinoma in situ aufgefasst $[8,23]$. In späteren Stadien traten noduläre oder papilläre Hyperplasien und Papillome mit oder ohne Atypien als Vorläufer von Karzinomen auf $[1,4,6$, 8, 10, 12, 14-16, 18, 22, 23].

Das durch BBN induzierte Spektrum der Neoplasien schien einigen Autoren identisch mit dem der menschlichen Harnblase zu sein $[15,18,23]$. Wenige Arbeiten legten das Augenmerk auf zytologische Veränderungen des Urothels [8, 12, 14]. Unter Berücksichtigung der Expositionsdauer ergab sich ein erheblicher Unterschied zwischen den früheren und den hier vorgelegten Ergebnissen. So wurden nach 20 oder 40 Wochen BBN Hyperplasie, Papillome und Karzinome, jedoch keine Dysplasie beschrieben [1, 10], nach 8 oder 20 Wochen eine fokale Hyperplasie [2, 9], Hyperplasie und $\mathrm{Pa}$ pillome nach 6 Wochen ohne und nach 12 Wochen mit fokaler Atypie [12].

Die Vielfalt und mangelnde Vergleichbarkeit der Diagnosen beruhte hauptsächlich auf dem Fehlen einer verbindlichen Klassifikation. Selbst in der Klinik bezeichneten die unterschiedlichen Begriffe wie dysplastische intraepitheliale Läsion, Dysplasie, Atypie, einfache und atypische Hyperplasie, Carcinoma in situ G1 und intraepitheliale Neoplasie dieselbe Urothelveränderung mit unklarer Prognose, die bei Tumorpatienten als Risikofaktor für ein Rezidiv aufgefasst wird [13]. Die WHO-Klassifikation trennt die urotheliale Hyperplasie von der Dysplasie. Die Hyperplasie weist keine zytologische Atypie und kein prämalignes Potential auf. Die Dysplasie zeigt zytologische und architektonische Veränderungen, die geringer ausgeprägt sind als die 
Tab. 1 Kernfläche $\left(\mu \mathrm{m}^{2}\right)$ : Maximum, Minimum, Mittelwert, Standardabweichung

\begin{tabular}{|c|c|c|c|c|c|c|c|c|c|c|c|}
\hline Woche & U.-Nr & $F_{\text {MIN }}$ & $F_{\text {MAX }}$ & $F_{\text {MIT }}$ & Fstdev & Woche & U.-Nr & $F_{\text {MIN }}$ & $F_{\text {MAX }}$ & $F_{\text {MIT }}$ & Fstdev \\
\hline Woche 0 & 3 & 37,9 & 240,3 & 76,2 & 27,6 & Woche 10 & 33 & 36,8 & 208,3 & 72,6 & 24,4 \\
\hline Woche 0 & 4 & 49,3 & 207,5 & 82,0 & 31,7 & Woche 10 & 34 & 37,5 & 97,7 & 69,2 & (1, 1 \\
\hline Woche 0 & 5 & 21,9 & 263,2 & 83,9 & 31,3 & 2 & 35 & 47,4 & 181,5 & 70,7 & 28,2 \\
\hline Woche 0 & 6 & 40,0 & 249,6 & 77,5 & 32,1 & Woche 12 & 36 & 39,1 & 258,6 & 76,1 & 34,9 \\
\hline Woche 0 & 7 & 39,1 & 173,0 & 67,9 & 17,2 & Woche 12 & 37 & 42,2 & 168,9 & 83,6 & 22,9 \\
\hline Woche 0 & 8 & 42,2 & 458,3 & 103,2 & 54,7 & 12 & 38 & 35,9 & 175,9 & 73,1 & 17,5 \\
\hline Woche 0 & 9 & 36,7 & 202,2 & 79,5 & 32,0 & Woc & 39 & 43,0 & 201,4 & 82,6 & 26,2 \\
\hline Woche 2 & 10 & 38,4 & 217,5 & 84,0 & 37,4 & Woche 12 & 40 & 33,9 & 242,6 & 74,2 & 29,8 \\
\hline Woche 2 & 11 & 44,5 & 170,0 & 78,6 & 24,8 & Woc & 41 & 47,0 & 24,3 & 80,2 & 16,3 \\
\hline Wo & 12 & 39,7 & 110,3 & 70,1 & 16,1 & 14 & 42 & 44,4 & 218,0 & 74,1 & 20,6 \\
\hline Woche 2 & 13 & 45,6 & 207,6 & 80,6 & 30,5 & Woc & 43 & 40,7 & 373,1 & 87,2 & 35,4 \\
\hline Woche 2 & 14 & 53,4 & 510,1 & 102,6 & 59,3 & Woche 14 & 44 & 53,1 & 210,2 & 86,8 & 22,1 \\
\hline Woche 4 & 15 & 57,0 & 329,2 & 93,8 & 39,1 & Woche 16 & 45 & 60,7 & 457,6 & 99,9 & 46,8 \\
\hline Wo & 16 & 48,4 & 370,7 & 96,9 & 38,9 & . & 46 & 47,3 & 156,2 & 78,0 & 21,0 \\
\hline Wo & 17 & 51,1 & 215,3 & 90,4 & 29,5 & Woc & 47 & 42,9 & 221,3 & 78,6 & 24,9 \\
\hline Woche 6 & 18 & 47,8 & 180,2 & 78,1 & 24,8 & Woche 16 & 48 & 40,6 & 15,9 & 68,4 & 14,6 \\
\hline Woche 6 & 19 & 45,6 & 131,0 & 73,3 & 16,4 & Woche 16 & 49 & 47,8 & 324,1 & 88,5 & 29,9 \\
\hline Woche 6 & 20 & 46,9 & 133,0 & 71,3 & 16,1 & Woc & 50 & 48,7 & 706,0 & 86,8 & 65,4 \\
\hline Wo & 2 & 55,9 & 22 & 80,8 & 25,4 & 8 & 5 & 55,7 & 9 & 84,5 & 18,2 \\
\hline Woche 6 & 22 & 40,6 & 156,3 & 78,2 & 20,4 & vrocn & 52 & 53,7 & 281,0 & 89,5 & 29,7 \\
\hline Woche 8 & 23 & 43,9 & 177,3 & 79,8 & 23,9 & Woche 18 & 53 & 44,2 & 156,5 & 81,0 & 21,4 \\
\hline Woche 8 & 24 & 45,0 & 171,6 & 78,1 & 21,9 & Woche 18 & 54 & 54,7 & 357,9 & 88,9 & 39,0 \\
\hline Wo & 25 & 40,3 & 173,1 & 79,6 & 22,1 & Woch & 55 & 35,8 & 146,5 & 73,7 & 26,4 \\
\hline Woche 8 & 26 & 35,6 & 232,6 & 73,6 & 25,4 & Woche 18 & 56 & 38,0 & 151,3 & 77,2 & 20,9 \\
\hline Woche 8 & 27 & 41,5 & 130,2 & 73,0 & 16,6 & Woche 20 & 57 & 38,4 & 198,0 & 70,9 & 24,4 \\
\hline Woche 8 & 28 & 40,5 & 131,2 & 74,1 & 16,0 & Woche 20 & 58 & 52,9 & 190,1 & 77,0 & 17,7 \\
\hline Woche 10 & 29 & 21,1 & 189,2 & 81,9 & 24,9 & Woche 20 & 59 & 42,2 & 127,7 & 77,1 & 15,6 \\
\hline Woche 10 & 30 & 30,1 & 212,1 & 74,2 & 27,8 & Woche 20 & 60 & 40,1 & 226,0 & 79,1 & 25,2 \\
\hline Woche 10 & 31 & 56,0 & 219,7 & 80,3 & 19,4 & Woche 20 & 61 & 27,7 & 158,7 & 65,3 & 18,2 \\
\hline Woche 10 & 32 & 43,0 & 283,5 & 79,1 & 31,5 & Woche 20 & 62 & 42,8 & 201,9 & 78,9 & 23,6 \\
\hline
\end{tabular}

des Carcinoma in situ. Primäre Dysplasien sollen in seltenen Fällen zu Blasentumoren fortschreiten [20]. Über die morphologische Analogie hinaus sind Klinik und Experiment offensichtlich durch grundlegend unterschiedliche Konzepte getrennt. Selbst auf der Grundlage der WHO-Klassifikation waren Hyperplasie und Dysplasie in der Frühphase des vorliegenden Experiments morphologisch nicht zweifelsfrei zu trennen.

Erste Veränderungen des Urothels, die histologisch als Hyperplasie zu deuten waren, traten in Woche 2 auf. Ab Woche 4 kam es zu Kernvergrößerungen, die in Woche 6 histologisch von einer Schichtungsstörung des Urothels begleitet wurden. Die Karyometrie stützte diese morphologischen Beobachtungen signifikant. Berücksichtigte man die bereits in Woche 4 aufgetretenen, morphologisch und morphometrisch fassbaren Veränderungen, waren diese frühen Alterationen des Urothels bereits als leichtgradige Dysplasie anzusehen.

Das hier vorgestellte Versuchsmodell wurde in den letzten Jahren zu genetischen und molekularpathologischen Untersuchungen herangezogen. So wurden p53- und H-ras-Mutationen, erhöhte Werte des „epidermal growth factor receptor" (EGFR) und Allelenverlust wie bei klinischen Tumoren und auch chromosomale Alterationen in frühen, nichtinvasiven Stadien beschrieben [23].

Tab. 2 Kernfläche: Klassen und Anzahl pro Intervall

\begin{tabular}{|c|c|c|c|c|c|c|c|c|c|c|c|}
\hline Klasse & 1 & 2 & 3 & 4 & 5 & 6 & 7 & 8 & 9 & 10 & $n$ \\
\hline$\left(\mu \mathrm{m}^{2}\right)$ & $0-40$ & $40-50$ & $50-60$ & $60-70$ & $70-80$ & 80-90 & $90-110$ & $110-150$ & $150-240$ & $>240$ & \\
\hline Woche 0 & 6 & 19 & 83 & 193 & 141 & 62 & 24 & 51 & 18 & 3 & 600 \\
\hline Woche 2 & 2 & 12 & 88 & 129 & 101 & 48 & 34 & 59 & 25 & 2 & 500 \\
\hline Woche 4 & 0 & 1 & 14 & 34 & 71 & 67 & 59 & 30 & 22 & 2 & 300 \\
\hline Woche 6 & 0 & 8 & 76 & 138 & 126 & 80 & 37 & 26 & 9 & 0 & 500 \\
\hline Woche 8 & 1 & 21 & 90 & 143 & 139 & 85 & 84 & 31 & 6 & 0 & 600 \\
\hline Woche 10 & 8 & 25 & 84 & 143 & 140 & 95 & 67 & 26 & 11 & 1 & 600 \\
\hline Woche 12 & 6 & 39 & 88 & 153 & 117 & 80 & 59 & 39 & 17 & 2 & 600 \\
\hline Woche 14 & 0 & 8 & 40 & 70 & 88 & 80 & 88 & 20 & 5 & 1 & 400 \\
\hline Woche 16 & 0 & 15 & 54 & 124 & 131 & 122 & 102 & 40 & 8 & 4 & 600 \\
\hline Woche 18 & 4 & 25 & 65 & 93 & 122 & 116 & 119 & 45 & 8 & 3 & 600 \\
\hline Woche 20 & 7 & 37 & 88 & 130 & 144 & 94 & 74 & 18 & 8 & 0 & 600 \\
\hline
\end{tabular}




\section{Schlussfolgerungen}

Die quantitative karyometrische Analyse führte in Kombination mit den zytologischen und histologischen Befunden zu einer objektiven Einordnung des zeitlichen Verlaufs der experimentellen, BBN-induzierten urothelialen Präkanzerose. Eine Dysplasie des Urothels wurde bereits nach einer 4-wöchigen Kanzerogenexposition fassbar und somit zeitlich wesentlich früher als auf der Grundlage vorausgegangener Untersuchungen anzunehmen war. Ergänzende, molekularpathologische Untersuchungen auf dem Gebiet der Entwicklung der experimentellen Präkanzerose gewannen mit den vorgelegten Befunden eine neue, verlässliche Basis.

\section{Korrespondenzadresse}

\section{PD Dr. H. H. Dahm}

Institut für Pathologie

Hirschlandstr. 97, 73730 Esslingen, Deutschland hhdahm@espath.de

Danksagung. Die Autoren danken Frau M. de Lede für hervorragende Assistenz bei allen Arbeitsschritten des Experiments.

\section{Einhaltung ethischer Richtlinien}

Interessenkonflikt. H. H. Dahm, V. Lehnen-Holtum und H. Rübben geben an, dass kein Interessenkonflikt besteht.

Alle nationalen Richtlinien zur Haltung und zum Umgang mit Labortieren wurden eingehalten, und die notwendigen Zustimmungen der zuständigen Behörden liegen vor.

Open Access. This article is distributed under the terms of the Creative Commons Attribution 4.0 International License (http://creativecommons.org/ licenses/by/4.0/), which permits unrestricted use, distribution, and reproduction in any medium, provided you give appropriate credit to the original author(s) and the source, provide a link to the Creative Commons license, and indicate if changes were made.

\section{Literatur}

1. Akagi G, Akagi A, Kimura Met al (1973) Comparison of bladder tumors induced in rats and mice with N-butyl-N-(4-hydroxybutyl)nitrosamine. Gan 64:331-336

2. Arai M, Kani T, Sugihara S et al (1974) Scanning and transmission electron microscopy of changes in the urinary bladder in rats treated with N-butyl-N(4-hydroxybutyl)-nitrosamine. Gan 65:529-540

3. Bjelkenkrantz G, Herder A, Gröntoft O et al (1982) Cytophotometric characterization of the WHO grades of transitional cell neoplasms. Path Res Pract 174:68-77

4. Cohen SM, Jacobs JB, Arai A et al (1976) Early lesions in experimental bladder cancer: experimental design and light microscopic findings. Cancer Res 36:2508-2511

5. Fukushima S, Hirose M, Tsuda H et al (1976) Histological classification of urinary bladder cancers in rats induced by N-butyl-N-(4-hydroxybutyl)nitrosamine. Gan 67:81-90

6. Fukushima S, Murasaki G, Hirose M et al (1982) Histopathological analysis of preneoplastic changes during N-butyl-N-(4-hydroxybutyl)nitrosamineinduced urinary bladder carcinogenesis in rats. Acta Pathol Jpn 32:243-250

7. Helander E, Hofer P-A, Holmberg G (1984) Karyometric investigations on urinary bladder carcinoma, correlated to histopathological grading. Virchows Arch A Pathol Anat Histopathol 403:117-125

8. Hicks RM, Chowaniec J (1978) Experimental induction, history, and ultrastructure of hyperplasia and neoplasia of the urinary bladder epithelium. Int Rev Exp Pathol 18:199-280

9. Ito N (1976) Early changes caused by N-butyl$\mathrm{N}$-(4-hydroxybutyl)nitrosamine in the bladder epithelium of different animal species. Cancer Res 36:2528-2531

10. Ito N, Arai M, Sugihara S et al (1975) Experimental urinary bladder tumors induced by $\mathrm{N}$-butyl$\mathrm{N}$-(4-hydrobutyl)-nitrosamine. In: Odashima S, Takayama S, Sato H (Hrsg) GANN monograph on cancer research. University Park, Baltimore, $S$ 367-381

11. Ito N, Hiasa Y, Tamai A et al (1969) Histogenesis of urinary bladder tumors induced by N-butyl$\mathrm{N}$-(4-hydroxybutyl)-nitrosamine in rats. Gan 60:401-410

12. Kunze E, Schauer A, Schatt S (1976) Stages of transformation in the development of N-Butyl-N(4-hydroxybutyl)nitrosamine-induced transitional cell carcinomas in the urinary bladder of rats. Z Krebsforsch Klin Onkol Cancer Res Clin Oncol 87:139-160

13. Murphy WM, Grignon DJ, Perlman EJ (2004) Tumors of the kidney, bladder, and related urinary structures. AFIP Atlas of tumor pathology, fourth series. American Registry of Pathology, Washington, DC, S280-283

14. Murphy WM, Irving CC (1981) The cellular features of developing carcinoma in murine urinary bladder. Cancer 47:514-522

15. Oliveira PA, Adega F, Palmeira CA et al (2007) DNA study of bladder papillary tumors chemically induced by N-butyl-N-(4-hydroxybutyl)nitrosamine in Fisher rats. Int J Exp Path 88:39-46

16. Oliveira PA, Palmeira C, Lopez CA (2005) Evaluation of DNA content in preneoplastic changes of mouse urinary bladder induced by N-butyl-N-(4hydroxybutyl)nitrosamine. J Exp Clin Cancer Res 24:609-616

17. Ooms ECM, Kurver PJH, Veldhuizen RW et al (1983) Morphometric grading of bladder tumors in comparison with histologic grading by pathologists. Hum Pathol 14:144-150

18. Palmeira C, Oliveira PA, Arantes-Rodrigues $R$ et al (2009) DNA cytometry and kinetics of rat urothelial lesions during chemical carcinogenesis. Oncol Rep 21:247-252

19. Rübben H, Dahm HH, Lutzeyer W (1982) Intravesicale Chemorezidivprophylaxe - eine randomisierte tierexperimentelle Studie. Helv Chir Acta 49:353-356
20. Sauter G, Algaba F, Amin MB et al et al (2004) Noninvasive urothelial tumors. In: Eble JN, Sauter G, Epstein Jl (Hrsg) Pathology and genetics of tumors of the urinary system and male genital organs. IARC, Lyon, S 111-112

21. Stenius F (1922) Studien über Pathologie und Klinik der Papillome und Karzinome der Harnblase. Arbeiten aus dem Pathol Inst der Universität Helsingfors N.F., Bd. 3., S27-190

22. Tiltman AJ, Friedell GH (1971) The histogenesis of experimental bladder cancer. Invest Urol 9:218-226

23. Vasconcelos-Nobrega C, Colaco A, Lopes C et al (2012) Review: BBN as an urothelial carcinogen. In Vivo (Brooklyn) 26:727-740 